\title{
Layer-specific strain echocardiography may reflect regional myocardial impairment in patients with hypertrophic cardiomyopathy
}

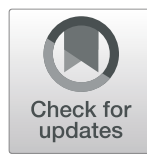

\author{
Zhongxiu Chen ${ }^{1}$, Chunmei Li ${ }^{1}$, Yajiao Li ${ }^{1}$, Li Rao ${ }^{1}$, Xiaoling Zhang ${ }^{1}$, Dan Long ${ }^{2}$ and Chen Li ${ }^{\text {* }}$
}

\begin{abstract}
Our study aimed to determine whether layer-specific strain (LSS) could reflect regional myocardial impairment in patients with hypertrophic cardiomyopathy (HCM). The study enrolled 50 patients with HCM and 30 age-matched healthy controls. Transmural gradient of longitudinal strain (TGLS), defined as the difference between the longitudinal strain of the endocardium and epicardium in a left ventricular segment, was used to reflect layerspecific myocardial impairment. Negative TGLS was consistently observed in healthy controls. The TGLS was relatively consistent within the basal, middle, and apical levels in healthy controls,but showed a significant gradient from the base towards the apex. In patients with HCM, the hypertrophic segments had significantly higher TGLS than the relatively normal segments or healthy controls at all 3 levels $(0.14 \% \pm 3.48 \%$ vs. $-2.65 \% \pm 4.44 \%$ vs. $-2.17 \% \pm 1.66 \%$ for basal, $-0.72 \% \pm 3.71 \%$ vs. $-4.02 \% \pm 4.00 \%$ vs. $-3.58 \% \pm 2.29 \%$ for middle, and $-8.69 \% \pm$ $7.96 \%$ vs. $-11.44 \% \pm 6.65 \%$ vs. $-10.04 \% \pm 3.20 \%$ for apex). Abnormal TGLS, defined as positive TGLS, in patients with HCM was associated with chest pain. In receiver operating characteristic curve analysis, a large area of abnormal TGLS (> 4 segments) had moderate accuracy for predicting chest pain (sensitivity, $73.3 \%$; specificity, $70.0 \%$ ). TGLS, a novel LSS derived parameter, may reflect regional myocardial impairment in patients with HCM.
\end{abstract}

Keywords: Layer-specific strain, Hypertrophic cardiomyopathy, Myocardial impairment, Microvascular dysfunction

\section{Key messages}

Patients with hypertrophic cardiomyopathy (HCM) could develop layer-specific myocardial impairment because of subendocardial microvascular dysfunction and focal cardiac fibrosis, however, this phenomenon has not received significant attention due to the lack of a feasible examination method.

The hypertrophic left ventricular segments had significantly higher TGLS, defined as the difference between territorial longitudinal strain of the endocardium and the epicardium, than the relatively normal segments of HCM patients or healthy controls.

\footnotetext{
*Correspondence: 69825160@qq.com

'Department of Cardiology, West China Hospital of Sichuan University, 37 Guo Xue Xiang, 610041 Chengdu, Sichuan, China

Full list of author information is available at the end of the article
}

Positive TGLS, which was consistently negative in healthy controls, was associated with chest pain in HCM patients, and may reflect endocardium-specific impairment of regional heart function in patients with HCM.

\section{Introduction}

Two-dimensional (2-D) speckle-tracking echocardiography is widely acknowledged as a sensitive method for evaluating global and regional left ventricular (LV) function. The ventricular wall consists of 3 layers, namely, the epicardium, mid-myocardium, and endocardium. Conventionally, assessment of the ventricular wall has not taken into account the different layers of the myocardium but has considered them as a single functional unit. This simplification generally works well. However, in patients with hypertrophic cardiomyopathy (HCM),

(C) The Author(s). 2021 Open Access This article is licensed under a Creative Commons Attribution 4.0 International License, which permits use, sharing, adaptation, distribution and reproduction in any medium or format, as long as you give appropriate credit to the original author(s) and the source, provide a link to the Creative Commons licence, and indicate if changes were made. The images or other third party material in this article are included in the article's Creative Commons licence, unless indicated otherwise in a credit line to the material. If material is not included in the article's Creative Commons licence and your intended use is not permitted by statutory regulation or exceeds the permitted use, you will need to obtain permission directly from the copyright holder. To view a copy of this licence, visit http://creativecommons.org/licenses/by/4.0/ The Creative Commons Public Domain Dedication waiver (http://creativecommons.org/publicdomain/zero/1.0/) applies to the data made available in this article, unless otherwise stated in a credit line to the data. 
this could be problematic. Previous studies have demonstrated that patients with HCM could develop subendocardial microvascular dysfunction [1] and focal cardiac fibrosis [2, 3]. These abnormalities could potentially lead to layer-specific myocardial impairment. However, the possibility of layer-specific myocardial impairment has not received significant attention due to the lack of a feasible examination method.

Improvements in the temporal and spatial resolution of 2-D echocardiography and strain analysis software have enabled layer-specific strain (LSS) analysis [4]. Several recent studies have reported predominant endocardial strain impairment in patients with subendocardial ischemia, proving the concept that LSS analysis could accurately reflect layer-specific myocardial impairment [5-8]. In the present study, we aimed to elucidate whether LSS could reflect layer-specific myocardial impairment in patients with HCM.

\section{Materials and Methods Study subjects}

This study prospectively recruited 67 consecutive patients with HCM according to the definition of the European Society of Cardiology Guidelines on diagnosis and management of HCM [9] at West China Hospital between March 2016 and January 2018. Patients with poor acoustic window (5 patients), LV ejection fraction (LVEF) $<50 \%$ (1 patient), history of septal myectomy (4 patients), atrial fibrillation (1 patient), obstructive coronary artery diseases (>50\% stenosis on either coronary angiography or computed tomography angiography, 3 patients) or valvular disease (3 patients) were excluded. Ultimately, 50 patients (mean age, $51.2 \pm 14.1$ years; $50 \%$ women) were enrolled. Thirty age-matched healthy controls with good acoustic windows were also recruited.

\section{Echocardiography}

All echocardiographic data assessments and image acquisitions were performed by an experienced sonographer by using an E9 ultrasonography system (GE Healthcare, Horten, Norway). Routine 2D images were acquired at a frame rate of 40-80 fps. LVEF was measured using the Simpson biplane method. The mitral $E / e^{\prime}-$ the ratio of mitral inflow early diastolic velocity (E) to the septal mitral annular spectral tissue Doppler early diastolic velocity $\left(e^{\prime}\right)$ - was also obtained in the apical 4-chamber view. Other parameters, such as maximum wall thickness, LV outflow tract gradient, and the phenomenon of systolic anterior motion, were also evaluated in accordance with the recommendations of the American Society of Echocardiography [10].
The 18-segment model, including posterior (basal, mid, and apical), anteroseptal (basal, mid, and apical), septal (basal, mid, and apical), lateral (basal, mid, and apical), inferior (basal, mid, and apical), and anterior (basal, mid, and apical) segments, was used in the segmental analysis. The LV segmental thickness in patients with $\mathrm{HCM}$ was measured to identify the hypertrophic $(>12 \mathrm{~mm})$ and normal $(\leq 12 \mathrm{~mm})$ segments, and dynamic images were observed. Three consecutive measurements were conducted to find the average value. Images in the apical 4-, 2-, and 3-chamber views from 3 consecutive cycles were stored digitally and analyzed offline.

\section{LV peak systolic longitudinal strain analyses}

The 2-D multilayer global strain in the LV myocardium was analyzed using ECHOPAC version 201 software (GE Healthcare). Aortic valve closing was automatically confirmed by software combined with manual adjustment. The border of the endocardium and the width of the target myocardium were manually traced and adjusted. The poorly traced segments (a total of 14 segments, mostly at the apical levels, of the $50 \mathrm{HCM}$ patients) were also excluded. The software automatically calculated segmental strain of the endocardial, middle, and epicardial myocardial layers (Figs. 1 and 2). The 2-D LV global longitudinal strain was measured from 3 apical views (apical 3-, 2-, and 4-chamber views). The transmural gradient of longitudinal strain (TGLS), a novel parameter defined as the difference between the territorial longitudinal strains of the endocardium and epicardium, was used to reflect endocardium-specific impairment.

\section{Patients' follow-up}

Patients were followed up for up to 6 months. Complete clinical follow-up was performed at 6 months, online or phone follow-up was performed at 1 and 3 months. Physicians were free to decide the management strategy during the follow-up period. During the follow-up, $28 \mathrm{HCM}$ patients received adjustment of drug regimens including adding or up-titration of beta blockers (22 patients), adding or up-titration of nondihydropyridine calcium channel blockers ( 3 patients) and other changes in regimens ( 3 patients). Both patients and healthy controls received another echocardiography examination at the end of the follow-up, or before any invasive treatment if invasive strategies including open surgery, septal alcohol ablation or pacemaker implantation were planned.

\section{Reproducibility of the TGLS-derived parameter}

The intra-observer and inter-observer reproducibility of TGLS was measured successively in 10 randomly 


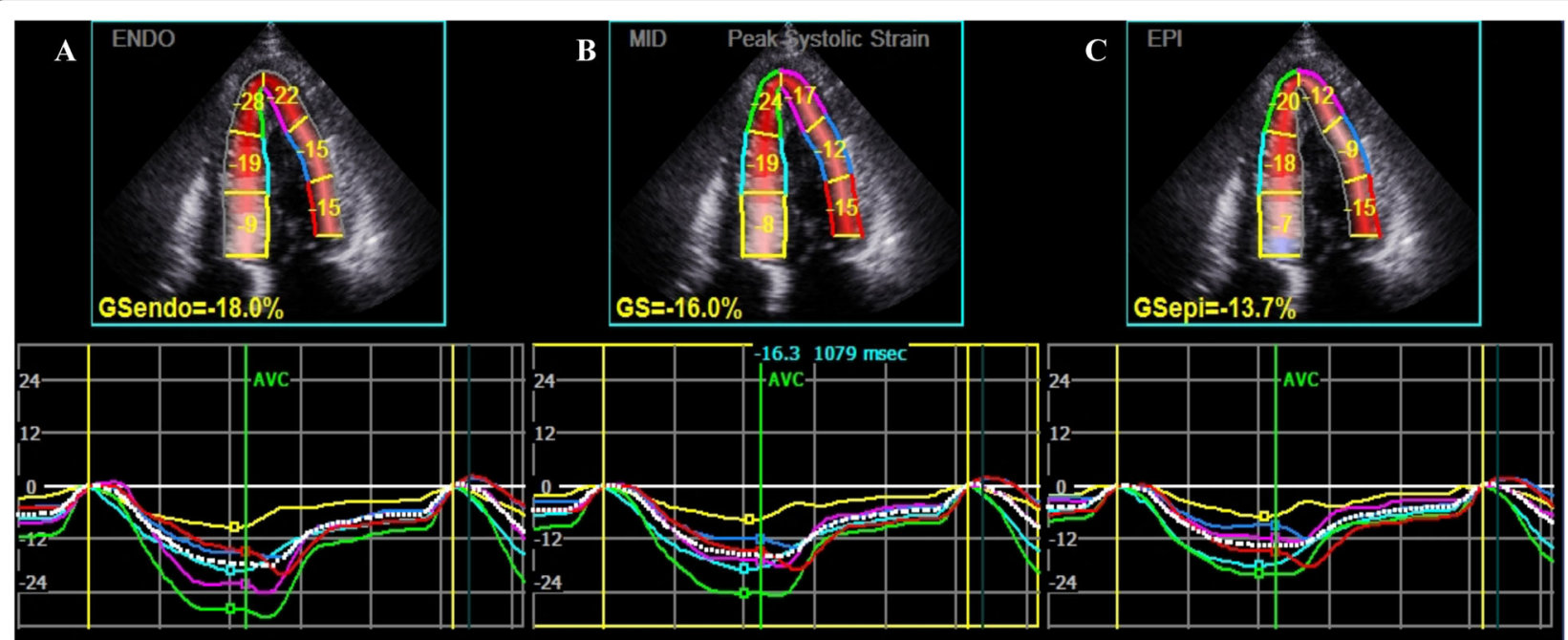

Fig. 1 Representative images of two-dimensional (2-D) left ventricular (LV) global layer-specific strain (LSS) analysis in a patient with hypertrophic cardiomyopathy (HCM) from apical 4-chamber views. The 2-D LV global LSS was also measured from apical 2- and 3-chamber views. The analyses were performed using Echo PAC version 201. The quantitative strain measurements of the endocardial (a), middle (b) and epicardial myocardial layers (c) were automatically calculated after the region of interest was manually traced and adjusted

selected patients with HCM. To test the intra-observer variability, a single observer analyzed the data twice on separate occasions at an interval of 1 month. To test the inter-observer variability, a second observer who was blinded to the first observer's measurements analyzed the same data.

\section{Statistical analyses}

All statistical analyses were conducted using SPSS version 19.0 (IBM Corporation, Armonk, NY, USA). The Shapiro-Wilk test was used to estimate the normality of the distributions of continuous data. Continuous variables with normal distributions are presented as mean \pm standard deviation, whereas those with skewed distributions are expressed as median (interquartile range). Baseline characteristics were compared between the HCM and control groups using independentsamples $t$ tests or the Wilcoxon test. Categorical variables were described as counts and percentages, and were compared using the Pearson chi-square test. Comparisons between the different segments, the 3 segmental levels in the controls, and the different subgroups were performed using analysis of variance. Multivariate ordinal logistic regression was used to assess association of different parameters with the grade of severity of abnormal TGLS segments. A receiver operating characteristic (ROC) curve was constructed for the completed model, and a probability threshold was selected for the

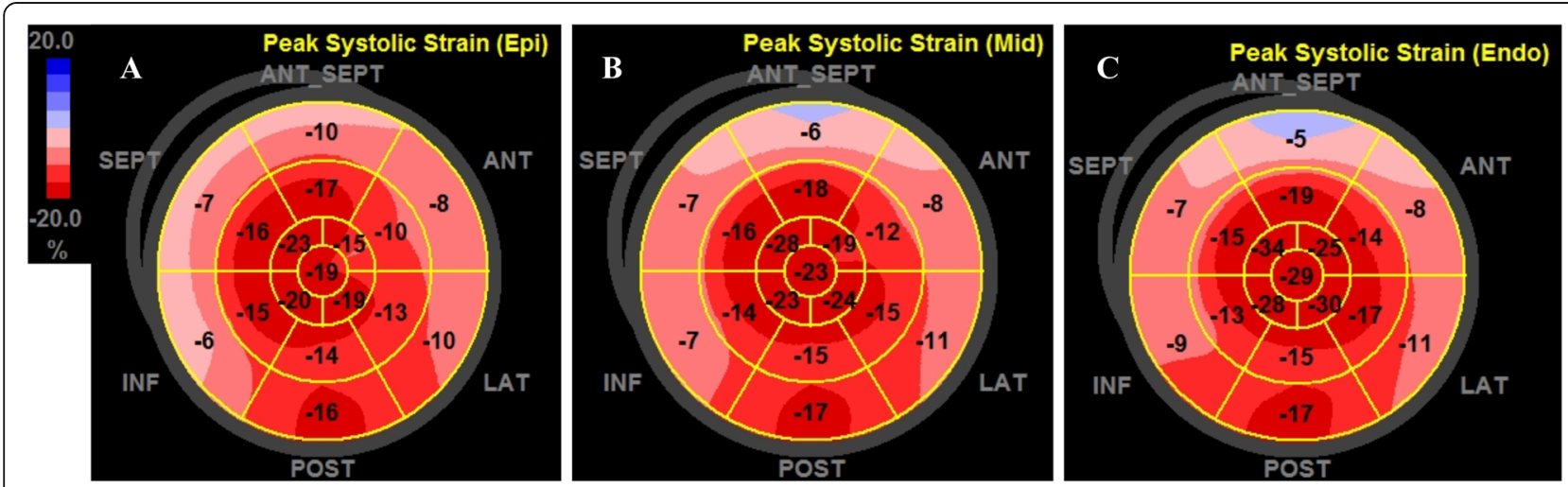

Fig. 2 Representative bull's-eye map of a patient with hypertrophic cardiomyopathy. A multilayer bull's-eye map, showing the epicardial (a), middle (b), and endocardial myocardial layers (c), was automatically generated after the layer-specific strain was measured in 3 apical views (apical 4-, 2-, and 3-chamber views). An 18-segment model was used in the segmental analysis 
likelihood of predicting chest pain based on the number of segments with abnormal TGLS. The resulting sensitivity and specificity were also estimated. The intraobserver and inter-observer variability values were analyzed using Bland-Altman bias plots, and by intraclass correlation coefficients (ICC). All tests were 2 -sided, and $\mathrm{p}<0.05$ was considered significant.

\section{Results}

\section{Study populations}

The baseline characteristics and clinical findings of the study participants are listed in Table 1 . Most of the
HCM patients had septal hypertrophy. The LV mass index was $109.5 \pm 35.7 \mathrm{~g} / \mathrm{m}^{2}$ in HCM group and $68.5 \pm$ $10.4 \mathrm{~g} / \mathrm{m}^{2}$ in the control group. $64 \%$ of the HCM patients had left ventricular outflow tract (LVOT) obstruction, with a mean resting gradient of $56 \mathrm{mmHg}$.

\section{Echocardiographic findings}

The layer specific longitudinal strain of healthy volunteers and HCM patients were listed in Table 2. In general, the longitudinal strain of endocardium, midmyocardium and epicardium were lower in the HCM patients than in the healthy controls. In the healthy

Table 1 Baseline characteristics of the study population

\begin{tabular}{|c|c|c|c|}
\hline Variables & $\mathrm{HCM}(\mathrm{n}=50)$ & Control $(n=30)$ & $\mathrm{p}$ value \\
\hline Age, y & $51.2 \pm 14.1$ & $49.3 \pm 10.8$ & 0.968 \\
\hline Female, n (\%) & $25(50.0)$ & $14(46.7)$ & 0.407 \\
\hline Asymmetric septal hypertrophy & $48(96 \%)$ & / & / \\
\hline Apical hypertrophy & $2(4 \%)$ & / & / \\
\hline LVOT obstruction & $32(64 \%)$ & / & / \\
\hline Systolic blood pressure, $\mathrm{mmHg}$ & $120.3 \pm 15.2$ & $119.4 \pm 16.9$ & 0.561 \\
\hline Diastolic blood pressure, $\mathrm{mmHg}$ & $72.8 \pm 10.9$ & $71.7 \pm 11.5$ & 0.838 \\
\hline Heart rate, bpm & $72.9 \pm 11.3$ & $76.9 \pm 10.4$ & 0.086 \\
\hline Body surface area, $\mathrm{m}^{2}$ & $1.69 \pm 0.20$ & $1.63 \pm 0.31$ & 0.989 \\
\hline Body mass index, $\mathrm{kg} / \mathrm{m}^{2}$ & $24.8 \pm 3.1$ & $23.5 \pm 4.3$ & 0.893 \\
\hline Hemoglobin, g/L & $135.5 \pm 17.4$ & $133.9 \pm 19.0$ & 0.865 \\
\hline NT-pro BNP, pg/ml & $1059.0(515.0-2575.0)$ & / & / \\
\hline hs-cTnT, ng/L & $22.4(13.4-45.8)$ & / & / \\
\hline Creatinine, $\mu \mathrm{mol} / \mathrm{L}$ & $74.1 \pm 17.7$ & $72.4 \pm 18.6$ & 0.869 \\
\hline$\beta$-blocker, n (\%) & $38(76)$ & / & / \\
\hline LA diameter, mm & $43.8 \pm 6.5$ & $31.3 \pm 4.7$ & 0.013 \\
\hline $\mathrm{LA}$ volume index, $\mathrm{ml} / \mathrm{m}^{2}$ & $34.6 \pm 12.8$ & $25.7 \pm 7.1$ & $<0.001$ \\
\hline$E / e^{\prime}$ & $21.6 \pm 8.0$ & $13.6 \pm 11.7$ & 0.008 \\
\hline Maximum wall thickness, mm & $21.5(19.8-25.0)$ & $9.1 \pm 1.8$ & $<0.001$ \\
\hline LV mass index, $\mathrm{g} / \mathrm{m}^{2}$ & $109.5 \pm 35.7$ & $68.5 \pm 10.4$ & $<0.001$ \\
\hline Resting LVOT gradient, mmHg & $56.0(9.0-82.0)$ & $7.8 \pm 1.6$ & $<0.001$ \\
\hline Systolic anterior motion, n (\%) & $29(58)$ & / & / \\
\hline Pulmonary artery, mm & $22(21-25)$ & $20.8 \pm 2.3$ & 0.003 \\
\hline PASP, $m m H g^{a}$ & $33(26-40)(n=23)$ & / & / \\
\hline Cardiac output, L/min & $6.57 \pm 2.40$ & $5.56 \pm 1.72$ & 0.022 \\
\hline LVEF, \% & $72.7 \pm 6.1$ & $64.3 \pm 6.4$ & 0.084 \\
\hline Chest pain, n (\%) & $24(48)$ & / & / \\
\hline Heart failure, n (\%) & $41(82)$ & / & / \\
\hline
\end{tabular}

NT-pro BNP: N-terminal propeptide of B-type natriuretic peptide; hs-cTnT: high-sensitive cardiac troponin T; LA: left atrial; LVOT: left ventricular outflow tract; E/e': ratio of early mitral inflow velocity (E) to early diastolic velocity at the septal mitral annulus (e'); PASP: pulmonary arterial systolic pressure; LVEF: left ventricular ejection fraction. The reference values for NT-pro BNP, hs-cTnT, and creatinine are $0-88 \mathrm{pg} / \mathrm{ml}, 0-14 \mathrm{ng} / \mathrm{ml}$, and 37-110 $\mu \mathrm{mol} / \mathrm{L}$ respectively. ${ }^{a}$ : The PASP was estimated using maximal tricuspid regurgitation pressure gradient on the continuous-wave Doppler tracing by the modified Bernoulli equation with the addition of right atrial pressure. Right atrial pressure was estimated at $3 \mathrm{mmHg}$ if the inferior vena cava (IVC) was not dilated ( $\leq 2.1 \mathrm{~cm}$ ) and there was a $50 \%$ decrease in the diameter during inspiration; it was estimated at $8 \mathrm{mmHg}$ if the IVC was dilated with normal inspiratory collapse and at $15 \mathrm{mmHg}$ if the IVC was dilated and did not collapse with inspiration 
Table 2 Layer-specific strain (\%) in healthy controls and HCM patients

\begin{tabular}{|c|c|c|c|c|c|c|c|c|}
\hline \multirow[t]{2}{*}{ Segments } & \multicolumn{3}{|c|}{ LSS in healthy controls } & \multirow{2}{*}{$\begin{array}{l}\text { GLS in } \\
\text { healthy } \\
\text { patients }\end{array}$} & \multicolumn{3}{|c|}{ LSS in HCM patients } & \multirow{2}{*}{$\begin{array}{l}\text { GLS in HCM } \\
\text { patients }\end{array}$} \\
\hline & endo & mid & epi & & endo & mid & epi & \\
\hline basAntSept & $-18.56 \pm 3.99$ & $-18.14 \pm 3.43$ & $-17.72 \pm 3.22$ & $-18.12 \pm 3.45$ & $-7.63 \pm 5.05^{*}$ & $-7.65 \pm 4.66^{*}$ & $-7.71 \pm 4.41^{*}$ & $-7.66 \pm 4.63^{*}$ \\
\hline basSept & $-17.82 \pm 2.24$ & $-17.74 \pm 2.42$ & $-17.67 \pm 2.83$ & $-17.75 \pm 2.40$ & $-6.25 \pm 4.75^{*}$ & $-7.61 \pm 4.99^{*}$ & $-8.99 \pm 5.29^{*}$ & $-7.62 \pm 4.89^{*}$ \\
\hline baslnf & $-20.02 \pm 8.39$ & $-19.65 \pm 8.34$ & $-19.29 \pm 8.44$ & $-19.68 \pm 8.32$ & $-11.47 \pm 7.30^{*}$ & $-11.66 \pm 5.95^{*}$ & $-11.39 \pm 5.18^{*}$ & $-11.51 \pm 5.99^{*}$ \\
\hline basPost & $-22.41 \pm 4.02$ & $-21.59 \pm 3.71$ & $-20.77 \pm 3.57$ & $-21.56 \pm 3.68$ & $-16.23 \pm 9.19^{*}$ & $-15.07 \pm 8.09^{*}$ & $-13.62 \pm 7.32^{*}$ & $-14.97 \pm 8.03^{*}$ \\
\hline basLat & $-20.60 \pm 4.22$ & $-19.45 \pm 4.03$ & $-18.29 \pm 4.22$ & $-19.40 \pm 4.13$ & $-15.50 \pm 8.22^{*}$ & $-13.63 \pm 6.77^{*}$ & $-11.61 \pm 5.92^{*}$ & $-13.58 \pm 6.83^{*}$ \\
\hline asAnt & $-18.24 \pm 6.98$ & $-17.42 \pm 6.72$ & $-16.60 \pm 6.55$ & $-17.45 \pm 6.70$ & $-11.46 \pm 6.90^{*}$ & $-10.09 \pm 6.25^{*}$ & $-8.59 \pm 5.83^{*}$ & $-10.05 \pm 6.25^{*}$ \\
\hline midAntSept & $-23.28 \pm 5.24$ & $-21.36 \pm 4.44$ & $-19.45 \pm 3.80$ & $-21.30 \pm 4.48$ & $-16.76 \pm 7.48^{*}$ & $-14.26 \pm 6.14^{*}$ & $-12.22 \pm 5.24^{*}$ & $-14.41 \pm 6.22^{*}$ \\
\hline nidSept & $-22.29 \pm 3.90$ & $-21.13 \pm 3.07$ & $-19.98 \pm 3.08$ & $-21.11 \pm 3.11$ & $-10.37 \pm 5.50^{*}$ & $-10.99 \pm 4.90^{*}$ & $-11.48 \pm 4.60^{*}$ & $-10.95 \pm 4.90^{*}$ \\
\hline midlnf & $-22.35 \pm 9.17$ & $-21.04 \pm 8.74$ & $-19.74 \pm 8.43$ & $-21.07 \pm 8.78$ & $-11.73 \pm 5.57^{*}$ & $-12.38 \pm 4.61^{*}$ & $-12.84 \pm 4.26^{*}$ & $-12.32 \pm 4.64^{*}$ \\
\hline hidPost & $-24.41 \pm 4.26$ & $-22.04 \pm 3.72$ & $-19.67 \pm 3.42$ & $-22.14 \pm 3.70$ & $-13.55 \pm 7.56^{*}$ & $-12.32 \pm 6.30^{*}$ & $-10.95 \pm 5.47^{*}$ & $-12.27 \pm 6.31^{*}$ \\
\hline midLat & $-21.69 \pm 4.11$ & $-19.69 \pm 3.83$ & $-17.70 \pm 3.80$ & $-19.65 \pm 3.88$ & $-13.96 \pm 7.97^{*}$ & $-11.55 \pm 6.62^{*}$ & $-9.33 \pm 5.73^{*}$ & $-11.61 \pm 6.66^{*}$ \\
\hline midAnt & $-19.98 \pm 8.49$ & $-18.59 \pm 7.83$ & $-17.21 \pm 7.23$ & $-18.54 \pm 7.80$ & $-13.48 \pm 7.01^{*}$ & $-11.31 \pm 6.08^{*}$ & $-9.31 \pm 5.45^{*}$ & $-11.36 \pm 6.10^{*}$ \\
\hline apAntSept & $-28.92 \pm 7.05$ & $-23.82 \pm 5.52$ & $-18.73 \pm 4.47$ & $-23.80 \pm 5.55$ & $-23.79 \pm 13.96$ & $-16.38 \pm 9.73^{*}$ & $-11.57 \pm 7.27^{*}$ & $-17.25 \pm 10.20$ \\
\hline apSept & $-30.07 \pm 4.79$ & $-24.98 \pm 4.26$ & $-19.88 \pm 3.94$ & $-24.96 \pm 4.24$ & $-23.43 \pm 11.56^{*}$ & $-17.04 \pm 8.82^{*}$ & $-12.62 \pm 7.31^{*}$ & $-17.70 \pm 9.01^{*}$ \\
\hline aplnf & $-27.22 \pm 14.52$ & $-23.65 \pm 11.15$ & $-17.83 \pm 9.65$ & $-22.52 \pm 12.03$ & $-23.06 \pm 11.22$ & $-16.41 \pm 7.40^{*}$ & $-11.99 \pm 5.61^{*}$ & $-17.15 \pm 7.85$ \\
\hline apPost & $-28.35 \pm 5.92$ & $-23.39 \pm 4.85$ & $-18.42 \pm 4.01$ & $-23.41 \pm 4.80$ & $-19.19 \pm 12.58^{*}$ & $-13.49 \pm 8.89^{*}$ & $-9.45 \pm 6.87^{*}$ & $-13.56 \pm 9.48^{*}$ \\
\hline apLat & $-28.00 \pm 6.06$ & $-23.02 \pm 4.70$ & $-18.04 \pm 3.74$ & $-23.12 \pm 4.61$ & $-20.31 \pm 11.31^{*}$ & $-13.84 \pm 8.49^{*}$ & $-9.49 \pm 6.80^{*}$ & $-14.55 \pm 8.70^{*}$ \\
\hline apAnt & $-24.18 \pm 14.90$ & $-19.59 \pm 12.41$ & $-15.00 \pm 10.08$ & $-19.57 \pm 12.6$ & $-22.15 \pm 12.28$ & $-14.95 \pm 8.79$ & $-10.07 \pm 6.70^{*}$ & $-15.73 \pm 9.11$ \\
\hline Total & $-23.24 \pm 8.15$ & $-20.90 \pm 6.59$ & $-18.44 \pm 5.79$ & $-20.84 \pm 6.67$ & $-15.34 \pm 10.22^{*}$ & $-12.72 \pm 7.37^{*}$ & $-10.73 \pm 6.04^{*}$ & $-12.91 \pm 7.59^{*}$ \\
\hline
\end{tabular}

LSS layer-specific strain; GLS global longitudinal strain; HCM hypertrophic cardiomyopathy; bas basal; ap apical; Post posterior; AntSept anteroseptal; Sept septal; Lat lateral; Inf inferior; Ant anterior; endo endocardial; mid middle; epi epicardial. * $p<; 0.05$ compared with healthy controls

volunteers, a negative TGLS (defined as the difference between the territorial longitudinal strains of the endocardium and epicardium) was observed in almost all segments. The normal range of TGLS greatly varied among the different segments (Table 3). A significant TGLS gradient from the base toward the apex was observed (Fig. 3). However, the TGLS was relatively consistent within the basal, middle, and apical levels. In patients with HCM, LV segments were further divided into hypertrophic segments (diastolic thickness of any part within the segment $>12 \mathrm{~mm}$ ) and non-hypertrophic segments (maximum diastolic thickness of the entire segment $\leq 12 \mathrm{~mm}$ ). Among the segments, $55.5 \%$, $49.8 \%$ and $10.1 \%$ were hypertrophic at the basal, middle, and apical levels, respectively. The TGLS in the non-hypertrophic segments of patients with HCM was not different from that of healthy controls according to level (Table 4; Fig. 4). However, the TGLS in the hypertrophic segments was significantly higher than that in the non-hypertrophic segments at almost all levels.

Abnormal TGLS, defined as a positive TGLS, was observed in $1.1 \%$ (6 of 540) of the normal LV segments in healthy controls. In patients with HCM, 11.3\% (61 of 542 ) of the non-hypertrophic segments and $45.3 \%$ (156 of 344) of the hypertrophic segments had abnormal TGLS (Table 5.).

\section{Associated with LV hypertrophy and LVOT obstruction}

The association of different parameters with the severity of abnormal TGLS segments (defined as the number of abnormal TGLS segments) was assessed in a multivariate ordinal logistic regression. The results demonstrated that LV mass index positively correlated with the severity of abnormal TGLS. In particular, with the increase in LV mass index for every $1 \mathrm{~g} / \mathrm{m}^{2}$, the OR of abnormal TGLS segments increased by 1.022 times (95\%CI: 1.002-1.043, $p=0.032)$. The presence of LVOT obstruction was also strongly correlated with the severity of abnormal TGLS (OR 5.823, $95 \%$ CI: $1.045-32.464, p=0.044)$. Other parameters including age, sex, body mass index, width of pulmonary artery, and E/e' were not significantly associated with the level of abnormal TGLS segments (Table 6). 
Table 3 Transmural gradient of longitudinal strain (\%) in healthy controls and HCM patients

\begin{tabular}{llllll}
\hline Segments & \multicolumn{2}{c}{ TGLS in healthy controls } & & \multicolumn{2}{c}{ TGLS in HCM patients } \\
\cline { 2 - 3 } & Mean \pm SD & $95 \%$ Cl & & Mean \pm SD & $95 \%$ Cl \\
\hline basAntSept & $-1.97 \pm 1.49$ & -2.65 to -1.29 & $0.08 \pm 2.20$ & -0.68 to 0.81 \\
basSept & $-1.49 \pm 1.06$ & -1.97 to -1.01 & $2.73 \pm 2.65^{*}$ & 1.89 to 3.59 \\
basInf & $-2.16 \pm 1.81$ & -2.98 to -1.33 & $-0.08 \pm 3.93$ & -1.39 to 1.26 \\
basPost & $-1.91 \pm 1.42$ & -2.56 to -1.27 & $-2.61 \pm 4.41$ & -4.11 to -1.17 \\
basLat & $-2.82 \pm 2.03$ & -3.74 to -1.89 & $-3.90 \pm 4.10$ & -5.31 to -2.64 \\
basAnt & $-2.69 \pm 1.75$ & -3.49 to -1.89 & $-2.87 \pm 2.65$ & -3.77 to -2.05 \\
midAntSept & $-3.83 \pm 2.27$ & -4.86 to -2.80 & $-4.54 \pm 3.23$ & -5.69 to -3.43 \\
midSept & $-3.80 \pm 3.67$ & -5.47 to -2.13 & $1.11 \pm 2.58^{*}$ & 0.22 to 1.93 \\
midInf & $-3.38 \pm 1.79$ & -4.20 to -2.57 & & $1.11 \pm 3.42^{*}$ & -0.06 to 2.34 \\
midPost & $-4.73 \pm 2.11$ & -5.69 to -3.78 & $-2.60 \pm 3.83^{*}$ & -4.10 to -1.33 \\
midLat & $-4.10 \pm 1.85$ & -4.95 to -3.26 & $-4.64 \pm 3.74$ & -5.84 to -3.45 \\
midAnt & $-3.30 \pm 2.10$ & -4.25 to -2.34 & $-4.17 \pm 2.88^{*}$ & -5.18 to -3.16 \\
apAntSept & $-9.82 \pm 3.69$ & -11.49 to -8.14 & $-12.22 \pm 7.62$ & -15.36 to -9.54 \\
apSept & $-10.19 \pm 2.08$ & -11.14 to -9.24 & $-10.82 \pm 6.43$ & -12.90 to -8.67 \\
aplnf & $-10.49 \pm 3.41$ & -12.09 to -8.90 & $-11.06 \pm 7.24$ & -13.69 to -8.46 \\
apPost & $-9.93 \pm 2.87$ & -11.23 to -8.62 & $-9.41 \pm 7.22$ & -12.43 to -6.58 \\
apLat & $-9.96 \pm 3.60$ & -11.60 to -8.32 & $-10.82 \pm 6.14$ & -13.02 to -8.82 \\
apAnt & $-9.90 \pm 3.64$ & -11.60 to -8.19 & $-12.07 \pm 6.84$ & -14.57 to -9.84 \\
Total & $-5.33 \pm 4.20$ & -5.76 to -4.91 & $-4.61 \pm 6.72$ & -5.18 to -4.12 \\
\hline
\end{tabular}

TGLS transmural gradient of longitudinal strain; HCM hypertrophic cardiomyopathy; SD standard deviation; $C l$ confidence interval; bas basal; ap apical; Post posterior; AntSept anteroseptal; Sept septal; Lat lateral; Inf inferior; Ant anterior. ${ }^{*} p<0.05$ compared with healthy controls

\section{Association with symptoms}

To further elucidate the clinical significance of abnormal TGLS, patients with HCM were further divided into 2 groups according to symptom. Patients with chest pain generally had more LV segments with abnormal TGLS than those without chest pain $(5.4 \pm 1.4$ vs. $3.5 \pm 1.7, \mathrm{p}=$ $0.001)$. In the ROC curve analysis, a large area of abnormal TGLS ( $>4$ segments) had moderate accuracy for predicting chest pain (sensitivity, $73.3 \%$; specificity, $70.0 \%$ ) (Fig. 5), while highly-sensitive cardiac troponin $\mathrm{T}$ and LVOT gradient did not significantly predict chest pain. Moreover, abnormal TGLS was not associated with heart failure, which was defined as current heart failure according to New York Heart Association class $\geq$ III, and elevated $\mathrm{N}$-terminal propeptide of B-type natriuretic peptide level.

\section{TGLS reflect treatment effect}

The changes in echocardiographic parameters during follow-up was summarized in Table 7 . The patients were further divided into two groups according to whether they received drug adjustment or not. In general, both conventional and strain derived parameters remained similar in healthy controls and patients who did not received drug adjustment. In patients who received adjustment of drug regimens, there is a slight decrease in LVOT pressure gradient, and decreased number of segments with abnormal TGLS.

\section{Reproducibility of TGLS}

As shown in Fig. 6, the intra-observer and inter-observer reproducibility of TGLS analysis was relatively good. The ICC for intra-observer and inter-observer analyses were 0.996 and 0.973 respectively.

\section{Discussion}

The principal findings of this study were as follows: (1) TGLS is a reproducible echocardiographic parameter. A negative TGLS was consistently observed in healthy controls. TGLS was significantly decreased from the base toward the apex but was relatively consistent within the basal, middle, and apical levels. (2) In patients with HCM, the hypertrophic LV segments had significantly higher TGLS than the relatively normal segments and healthy controls. (3) The abnormal TGLS in patients with HCM could be associated with subendocardial ischemia and LVOT pressure gradient.

Previous studies have demonstrated that strain imaging was helpful in diagnosis and prognosis prediction in $\mathrm{HCM}$ patients $[11,12]$. A significant difference between endocardial and epicardial strain has been reported since LSS was developed [4, 13]. Theoretically, strain imaging cannot distinguish the active contraction of the local myocardium and the passive stretching of adjacent layer. This could affect the sensitivity of strain imaging technique for subendocardial dysfunction. In an animal study, multi-layer strain imaging failed to show significant advantage over conventional one-layer strain imaging in detecting subendocardial ischemia [14]. However,the passive stretching effect was outweighed by active contraction in HCM patients since the myocardium was extremely thickened. Recently, Okada et al. [15] investigated HCM patients using LSS. They reported the global longitudinal strain was impaired in patients with HCM and the endocardium was more intensively affected. Interestingly, in this study the transmural gradient of segmental strain had moderate correlation with the segment wall thickness. A very recent large sample echocardiography study also confirmed this correlation [16]. In our study, we found that the transmural gradient of global longitudinal strain was similar in healthy controls and HCM patient. But TGLS was significantly higher in the hypertrophic segments of HCM than in segments with relatively normal thickness or healthy controls. 


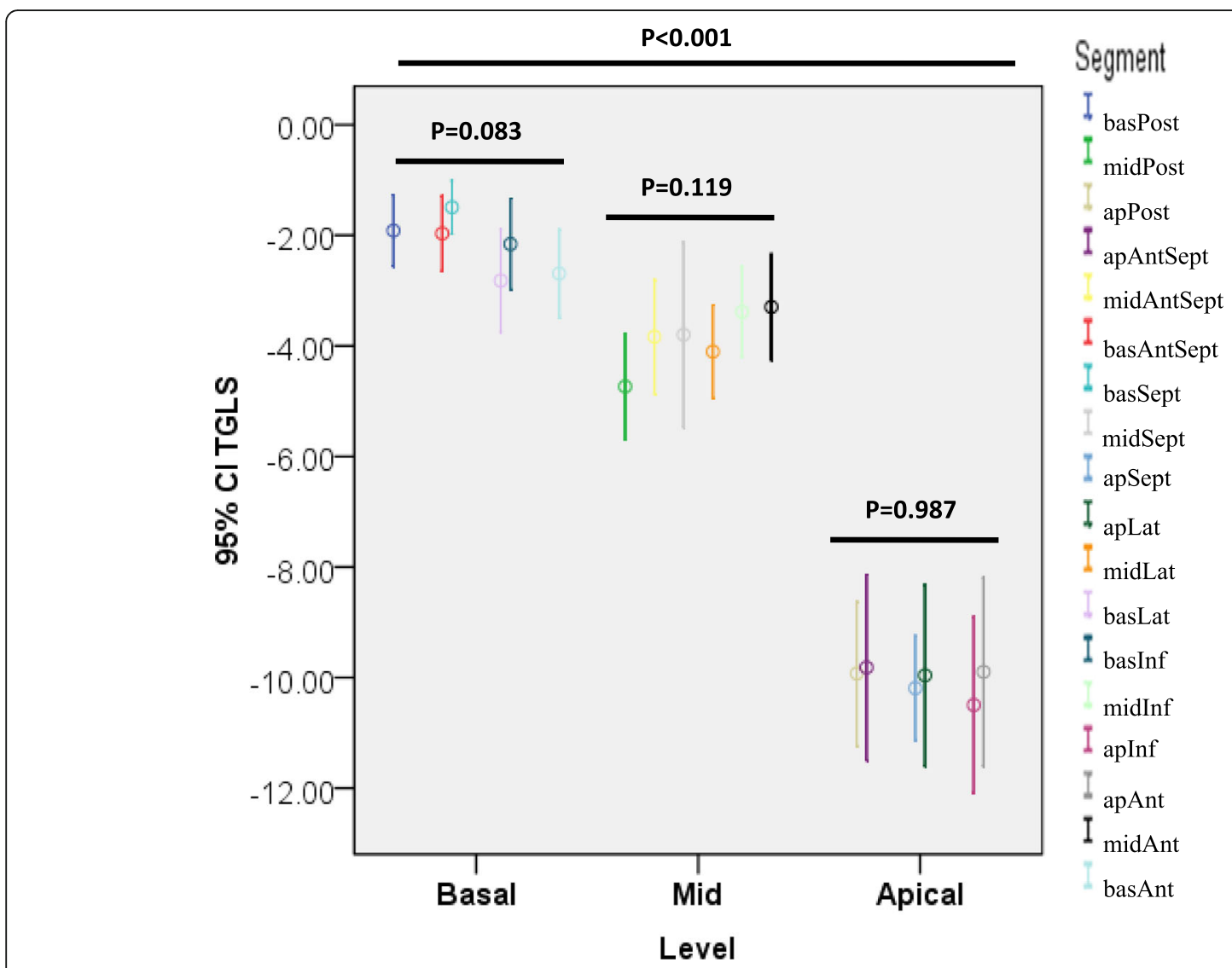

Fig. 3 In healthy volunteers, negative transmural gradient of longitudinal strain (TGLS) was observed in almost all segments. The normal range of TGLS greatly varied among the segments. However, the TGLS was relatively consistent within the basal, middle, and apical levels. A significant TGLS from the base toward the apex was observed. bas: basal; ap: apical; Post: posterior; AntSept: anteroseptal; Sept: septal; Lat: lateral; Inf: inferior; Ant: anterior

Although changes in transmural gradient of strain was observed in hypertrophic segments of HCM, its clinical significance has not been clearly elucidated yet. Recent echocardiography studies have reported that endocardial function was more affected in patients with ischemia [5, 6]. A very recent study has further demonstrated that subendocardial strain impairment was a sensitive parameter for early ischemia in dobutamine stressed echocardiography [17]. As a result, we hypothesized that abnormal TGLS was caused by subendocardial ischemia in the extremely hypertrophied myocardium. This hypothesis was supported by the multivariate ordinal logistic regression model demonstrating higher values of the LV mass index significantly and positively increase the likelihood of being in a higher level of abnormal TGLS segments, and by the ROC curve analysis showing large area of abnormal TGLS predicts the onset of chest pain but not heart failure.

Table 4 TGLS of healthy controls and patients with HCM with normal or hypertrophic segments

\begin{tabular}{llll}
\hline Levels & Healthy controls & $\begin{array}{l}\text { HCM patients } \\
\text { normal segments }\end{array}$ & $\begin{array}{l}\text { HCM patients } \\
\text { hypertrophic segments }\end{array}$ \\
\hline TGLS basal & $-2.17 \% \pm 1.66 \%$ & $-2.65 \% \pm 4.44 \%$ & $0.14 \% \pm 3.48 \% \%^{\mathrm{a}, \mathrm{b}}$ \\
& $(n=180)$ & $(n=133)$ & $-166)$ \\
TGLS mid & $-3.58 \% \pm 2.29 \%$ & $-4.02 \% \pm 4.00 \%$ & $-0.72 \% \pm 3.71 \% \%^{\mathrm{a}, \mathrm{b}}$ \\
& $(n=180)$ & $(n=150)$ & $(n=149)$ \\
TGLS apex & $-10.04 \% \pm 3.20 \%$ & $-11.44 \% \pm 6.65 \%$ & $-8.69 \% \pm 7.96 \%{ }^{\mathrm{c}}$ \\
& $(n=180)$ & $(n=259)$ & $(n=29)$ \\
\hline
\end{tabular}

TGLS transmural gradient of longitudinal strain; HCM hypertrophic cardiomyopathy. ${ }^{a} p<0.001$, compared with patients with $\mathrm{HCM}$ with normal segments. ${ }^{\mathrm{b}} p<$ 0.001 , compared with healthy controls. ${ }^{c} p<0.01$, compared with healthy controls; no significant difference was observed between the other two pairs. 


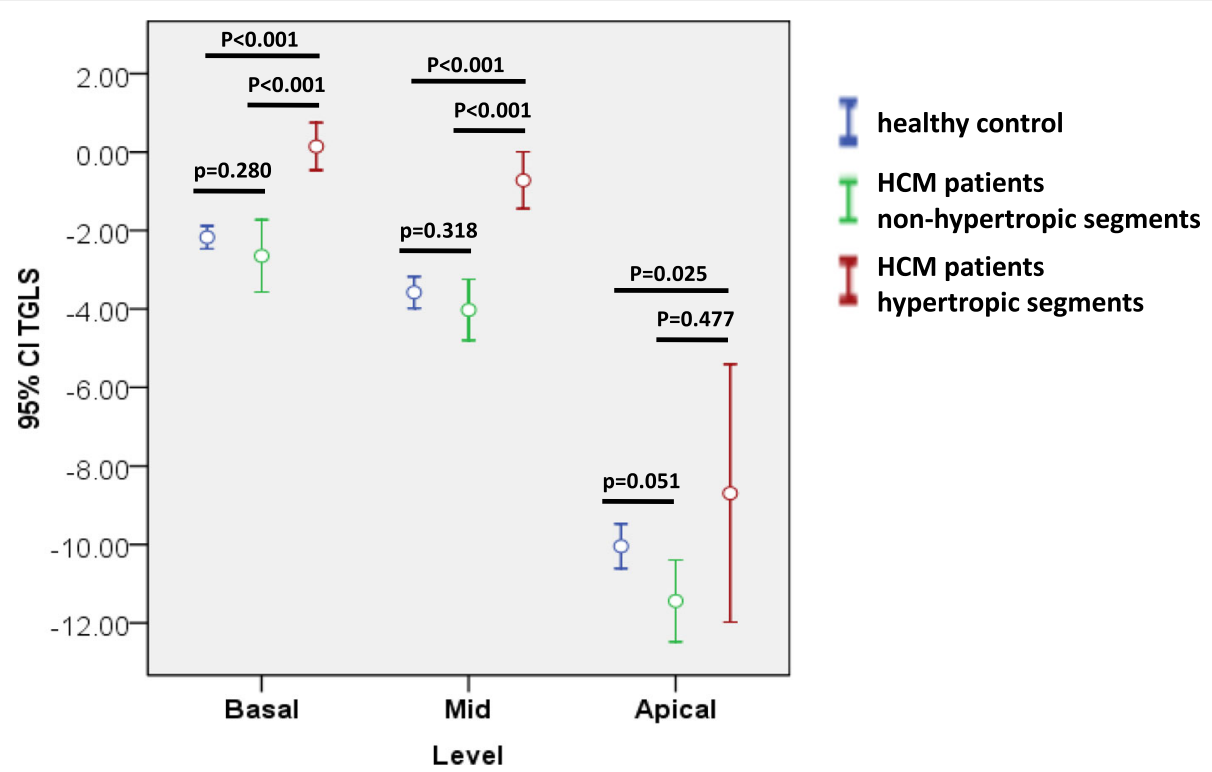

Fig. 4 The transmural gradient of longitudinal strain (TGLS) in the non-hypertrophic segments of patients with hypertrophic cardiomyopathy (HCM) was not significantly different from that of healthy controls according to level. However, the TGLS in the hypertrophic segments was significantly higher than that in the non-hypertrophic segments at almost all levels

Furthermore, a recent study reported improvement of subendocardial strain in patients with hypertrophic obstructive cardiomyopathy after alcohol septal ablation [18], suggesting the abnormality in transmural strain gradient may also be associated with the LVOT obstruction. In our study, nearly two thirds of the HCM patients had significant LVOT obstruction. In a multivariate logistic regression, we found that the severity of abnormal TGLS was associated with not only LV hypertrophy, but also LVOT obstruction. During a 6-month follow-up, $56 \%$ of the $\mathrm{HCM}$ patients receiving drug adjustment aimed to lower the LVOT pressure gradient. In these patients, both significant drop in LVOT pressure gradient and decrease in number of segments with abnormal TGLS were observed at the end of the follow-up. Meanwhile, in patients who maintained their regimens, both their LVOT pressure gradient and LSS parameters remained stable during the follow-up. This indicated that LVOT obstruction may contribute to the abnormal subendocardial strain in HCM patients. Interestingly, in two recent studies mainly including non-obstructive $\mathrm{HCM}$ patients, the prevalence of positive transmural gradient was very low $[15,16]$. These evidences also suggested the LVOT obstruction may contribute to the abnormal transmural strain gradient.

Another difficulty in interpreting the abnormal transmural strain gradient was the lack of universal measurement and normal reference value. Nagata et al. [19] attempted to determine the normal reference value of LSS in a relatively large number of healthy subjects. In that study, a significant transmural gradient of longitudinal strain from the endocardium toward the epicardium was observed. Nagata et al. suggested an endocardial strain to epicardial strain ratio to reflect this transmural strain gradient. The endocardial strain to epicardial strain ratio was a stable parameter in healthy

Table 5 Abnormal TGLS, defined as a positive TGLS, among healthy controls and patients with HCM with normal or hypertrophic segments

\begin{tabular}{lllll}
\hline Variables & Healthy controls & $\begin{array}{l}\text { HCM patients } \\
\text { normal segments }\end{array}$ & $\begin{array}{l}\text { HCM patients } \\
\text { hypertrophic segments }\end{array}$ \\
\hline TGLS $>0.0 \%, \mathrm{n}$ & 6 & 61 & 156 & $\boldsymbol{p}$ value \\
TGLS $\leq 0.0 \%, \mathrm{n}$ & 534 & 481 & 188 & $/$ \\
Ratio of $>0.0 \%$ & $1.1 \%(6 / 540)$ & $11.3 \%(61 / 542)$ & $45.3 \%(156 / 344)$ & $<0.001$ \\
\hline
\end{tabular}

TGLS transmural gradient of longitudinal strain; HCM hypertrophic cardiomyopathy. The chi-square test was performed for 2-group comparisons among the 3 groups. The $p$ values obtained in all the tests were $<0.001$. A $p$ value of $<0.017$ was assumed to be significant. 
Table 6 Predictors of the severity of abnormal TGLS

\begin{tabular}{|c|c|c|c|c|}
\hline & Coefficient B & SE & $P$ value & Adjusted OR $(95 \% \mathrm{Cl})$ \\
\hline Age & 0.043 & 0.030 & 0.154 & $1.044(0.984-1.107)$ \\
\hline Sex/Male & 0.174 & 0.712 & 0.807 & $1.190(0.295-4.808)$ \\
\hline $\mathrm{BMI}, \mathrm{kg} / \mathrm{m}^{2}$ & 0.064 & 0.124 & 0.605 & $1.066(0.837-1.358)$ \\
\hline $\mathrm{PA}, \mathrm{mm}$ & 0.116 & 0.106 & 0.275 & $1.123(0.912-1.382)$ \\
\hline LV mass index, $\mathrm{g} / \mathrm{m} 2$ & 0.022 & 0.010 & 0.032 & $1.022(1.002-1.043)$ \\
\hline LVOTO & 1.762 & 0.877 & 0.044 & $5.823(1.045-32.464)$ \\
\hline$E / e^{\prime}$ & 0.049 & 0.047 & 0.298 & $1.050(0.958-1.151)$ \\
\hline
\end{tabular}

$B M I$ body mass index; $C I$ confidence interval; E/e' ratio of early mitral inflow velocity (E) to early diastolic velocity at the septal mitral annulus (e'); $L V$ left ventricular; LVOTO left ventricular outflow tract obstruction; OR odds ratio; PA pulmonary artery; SE standard error

subjects. However, in patients with abnormal LV deformation, the denominator could be near to zero, which hampered the calculation. Under this circumstance, we believe that the TGLS, defined as the absolute difference in longitudinal strain between the endocardium and the epicardium in a certain LV segment, could be a more reliable parameter for layer-specific LV dysfunction (Fig. 7).

As for the normal reference value, we simply used $0.0 \%$ as the cutoff value for abnormal TGLS in the present study. This cutoff value had relatively high specificity. Healthy controls generally had a negative TGLS. The false-positive rate of TGLS was only $1.1 \%$ in healthy controls. In HCM patients, positive TGLS was found mainly in the hypertrophic segments. However, a significant change of TGLS from the basal toward the apical segments was observed in our study. This trend was also observed by other investigators. In the study by Nagata et al. [19], the average difference between the endocardial and epicardial longitudinal strain was $2.0 \%$ in the basal segments, $-3.4 \%$ in the middle segments, and $-11.4 \%$ in the apical segments. As a result, a

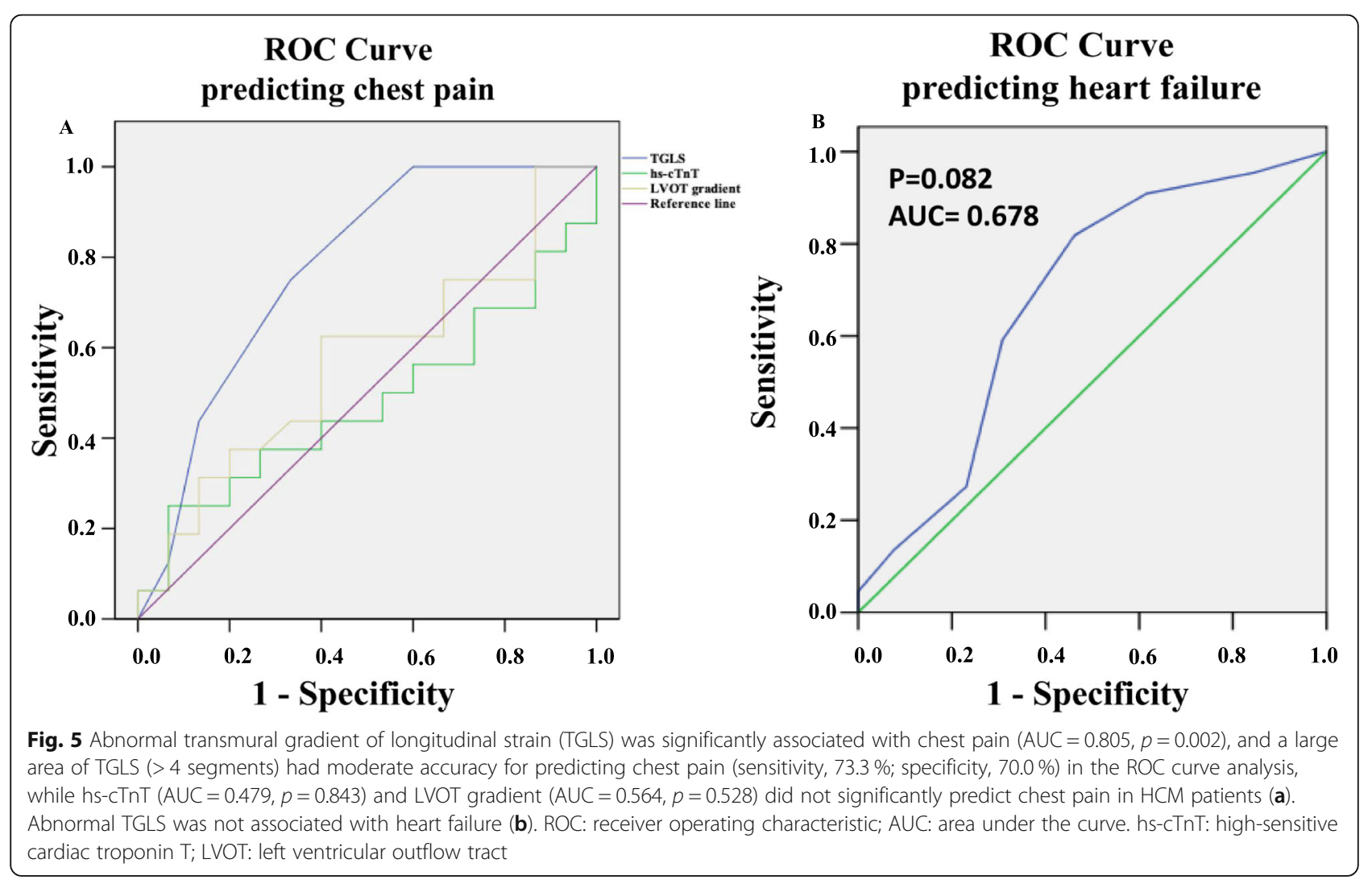


Table 7 TGLS in healthy controls and patients with or without drug adjustment

\begin{tabular}{|c|c|c|c|c|c|c|}
\hline & \multicolumn{2}{|c|}{ Healthy controls $(n=30)$} & \multicolumn{2}{|c|}{$\begin{array}{l}\text { Patients without drug } \\
\text { adjustment }(n=22)\end{array}$} & \multicolumn{2}{|c|}{$\begin{array}{l}\text { Patients received drug } \\
\text { adjustment }(n=28)\end{array}$} \\
\hline & Baseline echo & Follow-up echo & Baseline echo & Follow-up echo & Baseline echo & Follow-up echo \\
\hline LVEF, \% & $64.3 \pm 6.4$ & $65.6 \pm 5.7$ & $70.8 \pm 6.1$ & $69.6 \pm 7.3$ & $72.7 \pm 5.4$ & $73.7 \pm 6.5$ \\
\hline Resting LVOT gradient, mmHg & $7.8 \pm 1.6$ & $7.0 \pm 2.1$ & $18.2(6.1-30.3)$ & $18.0(6.0-30.0)$ & $70.0(47.0-90.0)$ & $40.0(17.0-68.0) *$ \\
\hline Global LS, \% & $-20.3 \pm 6.84$ & $-22.6 \pm 5.49$ & $-12.3 \pm 7.87$ & $-12.3 \pm 8.79$ & $-13.7 \pm 10.10$ & $-14.7 \pm 9.70$ \\
\hline TGLS basal, \% & $-2.17 \pm 1.66$ & $-2.58 \pm 1.03$ & $-1.22 \pm 2.10$ & $-1.19 \pm 2.70$ & $-1.01 \pm 2.14$ & $-1.71 \pm 2.98^{* *}$ \\
\hline TGLS mid, \% & $-3.58 \pm 2.29$ & $-3.27 \pm 2.88$ & $-3.28 \pm 2.48$ & $-2.98 \pm 2.39$ & $-2.72 \pm 2.50$ & $-3.31 \pm 2.64^{* *}$ \\
\hline TGLS apex, \% & $-10.54 \pm 3.20$ & $-11.10 \pm 4.21$ & $-9.9 \pm 3.99$ & $-9.5 \pm 3.75$ & $-9.8 \pm 4.11$ & $-10.21 \pm 3.79$ \\
\hline Number of segments with abnormal TGLS & 6 & 8 & 72 & 79 & 145 & $84^{* * *}$ \\
\hline
\end{tabular}

LVEF left ventricular ejection fraction; LVOT left ventricular outflow tract; $L S$ longitudinal strain; TGLS transmural gradient of longitudinal strain. ${ }^{*}, * * * * *: p<0.05$, $0.01,0.001$, compared with baseline data in patients received drug adjustment

level-specific cutoff value might increase the sensitivity. Future studies with larger sample size to determine the individualized TGLS cutoff value for the basal, middle, and apical levels, respectively, would be necessary. This is particularly important for the apical segments, where the normal range of TGLS was significantly lower than in the basal and middle segments. However, in our study, only a small proportion of HCM patients had apical hypertrophy. Furthermore, the relatively poor imaging quality of the apex also hampered accurate strain calculation. Thus, we observed a very wide confidence interval for apical TGLS in HCM patients. Future studies involving more patients with apical HCM might help determine the cutoff value for abnormal TGLS in the apical segments.

\section{Study limitation}

This study has several limitations. Firstly, the major limitation of the present study is the relatively small sample size. Secondly, the possibility that some patients with HCM might also have coronary artery disease but was not totally excluded. Most of the study subjects underwent coronary angiography or coronary computed tomography to rule out potential coronary artery disease. However, in some young and asymptomatic patients, it is unethical to perform invasive coronary angiography or coronary computed tomography. Thirdly, TGLS may be also affected by the presence of microvascular dysfunction in HCM patients. Thus, comparison of echocardiography derived strain with more sensitive perfusion techniques, such as nuclear imaging and CMR, could
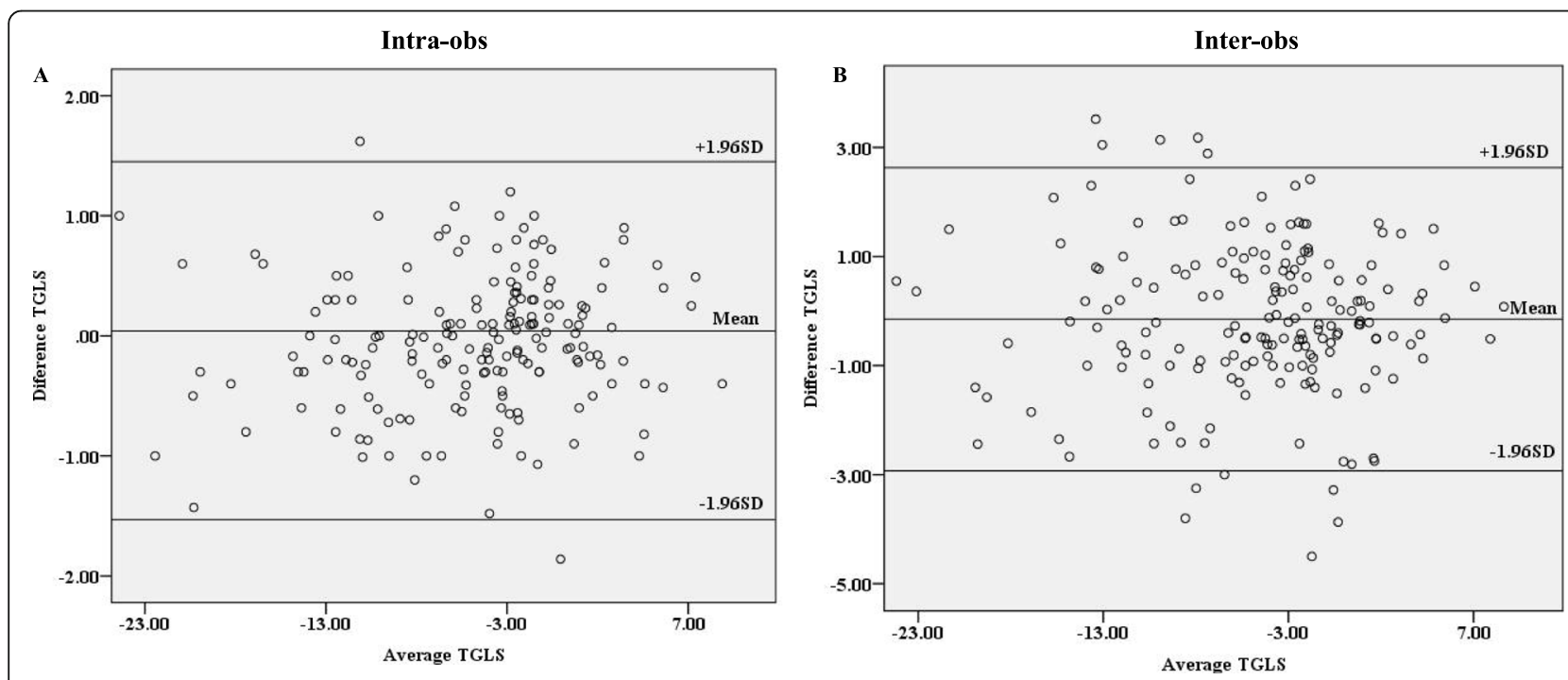

Fig. 6 Bland-Altman plots of the intra-observer and inter-observer reproducibility of the transmural gradient of longitudinal strain (TGLS). The limit of agreement was $-1.532-1.449 \%$ for the intra-observer comparison (a), and - 2.929-2.629\% for the inter-observer comparison (b) 

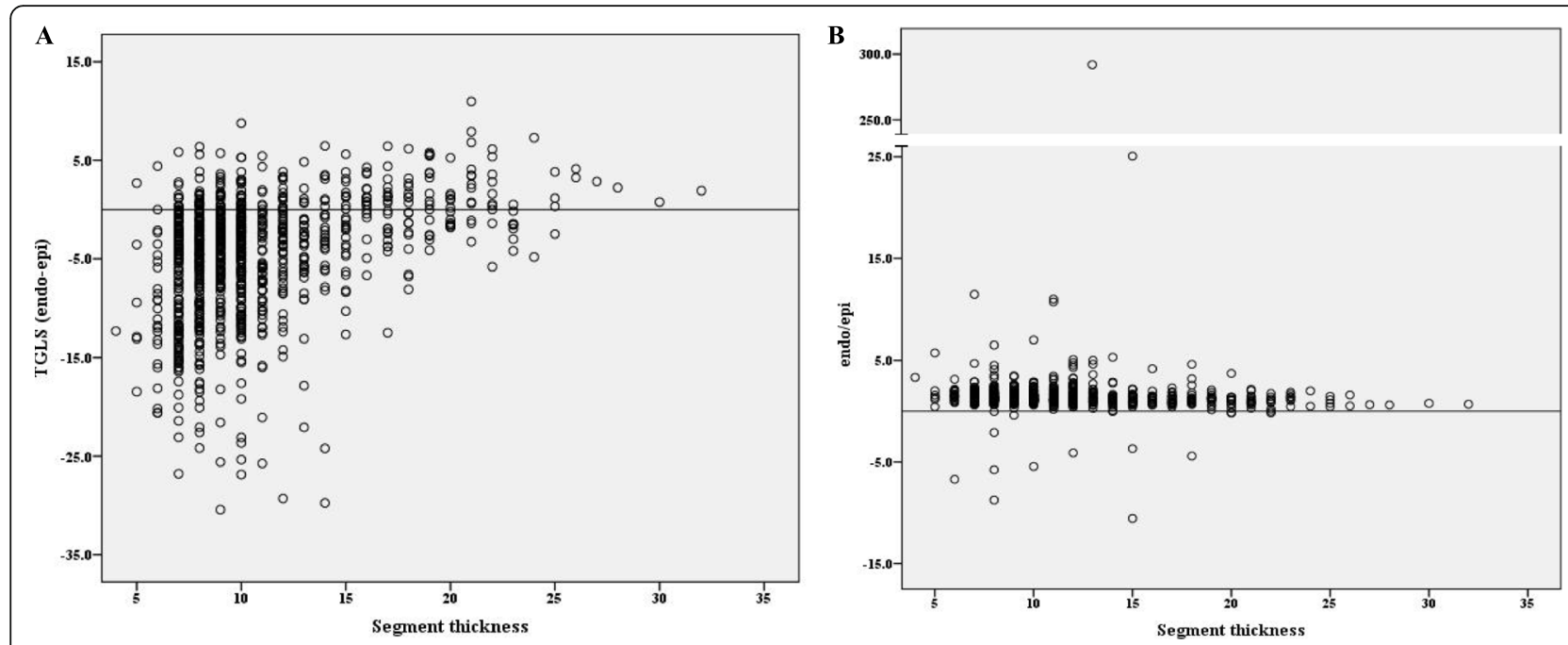

Fig. 7 Scatter plot of TGLS (a) and the ratio of endocardial strain and epicardial strain (b). Note that there's an abnormal extreme value in the ratio of endocardial strain and epicardial strain because the epicardial strain was near to zero $(-0.02 \%)$

provide more information. However, only a small portion of our patients received CMR or nuclide myocardial imaging. Thus, comparison of these techniques was not possible in this article. Further studies will be helpful to elucidate the mechanism underlying the layer specific impairment of myocardial strain.

\section{Conclusions}

A novel LSS-derived parameter named TGLS, defined as the difference between the territorial longitudinal strains of the endocardium and epicardium, could reflect the endocardium-specific impairment of regional heart function in patients with HCM.

\section{Acknowledgements}

The authors would like to thank Editage for assistance with language editing.

\section{Authors' contributions}

Zhongxiu Chen enrolled patients, directed the researches, carried out statistical analysis, and wrote the manuscript. Chunmei Li, Yajiao Li, Xiaoling Zhang, and Li Rao acquired and analyzed echocardiographic images. Dan Long followed up patients and collected data. Chen Li conceived, instructed, reviewed, and revised the manuscript. All authors read and approved the final manuscript.

\section{Funding}

This study was funded by grants from Key Research \& Development project of Sichuan Province (grant number 2018 SZ0018 and 2020YFS0244). The funders had no role in study design, data collection and analysis, decision to publish, or preparation of the manuscript.

\section{Availability of data and materials}

The regarding raw data and material of this manuscript can be available through the corresponding author by 69,825,160@qq.com if required.

\section{Ethics approval and Consent to participate}

The study protocol was approved by the ethics committee of the West China Hospital of Sichuan University (Sichuan, China). Written informed consent was obtained from all participants.
Consent for publication

Not applicable.

\section{Competing interests}

None.

\section{Author details}

'Department of Cardiology, West China Hospital of Sichuan University, 37 Guo Xue Xiang, 610041 Chengdu, Sichuan, China. ${ }^{2}$ Key Laboratory of Transplant Engineering and Immunology, West China Hospital of Sichuan University, High-tech Zone, Chengdu, Sichuan, China.

Received: 23 September 2020 Accepted: 10 February 2021

Published online: 03 March 2021

\section{References}

1. Petersen SE, Jerosch-Herold M, Hudsmith LE, et al. Evidence for microvascular dysfunction in hypertrophic cardiomyopathy: new insights from multiparametric magnetic resonance imaging. Circulation. 2007;115: 2418-25.

2. Galati G, Leone O, Pasquale F, et al. Histological and Histometric Characterization of Myocardial Fibrosis in End-Stage Hypertrophic Cardiomyopathy: A Clinical-Pathological Study of 30 Explanted Hearts. Circ Heart Fail. 2016;9:e003090

3. Iles LM, Ellims AH, Llewellyn $H$, et al. Histological validation of cardiac magnetic resonance analysis of regional and diffuse interstitial myocardial fibrosis. Eur Heart J Cardiovasc Imaging. 2015;16:14-22.

4. Adamu U, Schmitz F, Becker M, Kelm M, Hoffmann R. Advanced speckle tracking echocardiography allowing a three-myocardial layer-specific analysis of deformation parameters. Eur J Echocardiogr. 2009;10:303-8.

5. Sarvari SI, Haugaa KH, Zahid W, et al. Layer-specific quantification of myocardial deformation by strain echocardiography may reveal significant CAD in patients with non-ST-segment elevation acute coronary syndrome. JACC Cardiovasc Imaging. 2013;6:535-44.

6. Zhang L, Wu WC, Ma H, Wang H. Usefulness of layer-specific strain for identifying complex CAD and predicting the severity of coronary lesions in patients with non-ST-segment elevation acute coronary syndrome: Compared with Syntax score. Int J Cardiol. 2016;223:1045-52.

7. Park JH, Woo JS, Ju S, et al. Layer-specific analysis of dobutamine stress echocardiography for the evaluation of coronary artery disease. Medicine. 2016;95:e4549.

8. Yu W, Li SN, Chan GC, Ha SY, Wong SJ, Cheung YF. Transmural strain and rotation gradient in survivors of childhood cancers. Eur Heart J Cardiovasc Imaging. 2013;14:175-82. 
9. Authors/Task Force members. Elliott PM, Anastasakis A, et al. 2014 ESC Guidelines on diagnosis and management of hypertrophic cardiomyopathy: the Task Force for the Diagnosis and Management of Hypertrophic Cardiomyopathy of the European Society of Cardiology (ESC). Eur Heart J. 2014; 35: 2733-79.

10. Lang RM, Badano LP, Mor-Avi V, et al. Recommendations for cardiac chamber quantification by echocardiography in adults: an update from the American Society of Echocardiography and the European Association of Cardiovascular Imaging. J Am Soc Echocardiogr. 2015;28:1-39.

11. Haland TF, Saberniak J, Leren IS, Edvardsen T, Haugaa KH. Echocardiographic comparison between left ventricular non-compaction and hypertrophic cardiomyopathy. Int J Cardiol. 2017;228:900-5.

12. Tower-Rader A, Mohananey D, To A, Lever HM, Popovic ZB, Desai MY Prognostic Value of Global Longitudinal Strain in Hypertrophic Cardiomyopathy: A Systematic Review of Existing Literature. JACC Cardiovasc Imaging. 2019;12:1930-42.

13. Hashimoto I, Li X, Hejmadi Bhat A, Jones M, Zetts AD, Sahn DJ. Myocardial strain rate is a superior method for evaluation of left ventricular subendocardial function compared with tissue Doppler imaging. J Am Coll Cardiol. 2003;42:1574-83.

14. Moen CA, Salminen PR, Dahle GO, Hjertaas JJ, Grong K, Matre K. Multi-layer radial systolic strain vs. one-layer strain for confirming reperfusion from a significant non-occlusive coronary stenosis. Eur Heart J Cardiovasc Imaging. 2013;14:24-37.

15. Okada K, Yamada S, Iwano H, et al. Myocardial shortening in 3 orthogonal directions and its transmural variation in patients with nonobstructive hypertrophic cardiomyopathy. Circ J. 2015;79:2471-9.

16. Huang MS, Lee WH, Tsai HR, Liu YW, Liu PY, Tsai WC. Value of layer-specific strain distribution patterns in hypertrophied myocardium from different etiologies. Int J Cardiol. 2019;281:69-75.

17. Dessalvi CC, Deidda M, Farci S, Longu G, Mercuro G. Early ischemia identification employing $2 \mathrm{D}$ speckle tracking selective layers analysis during dobutamine stress echocardiography. Echocardiography. 2019;36:2202-8.

18. Zhang J, Zhu L, Jiang X, Hu Z. Layer-specific strain analysis of left ventricular myocardium after alcohol septal ablation for hypertrophic obstructive cardiomyopathy. Medicine. 2018;97:e13083.

19. Nagata Y, Wu VC, Otsuji Y, Takeuchi M. Normal range of myocardial layerspecific strain using two-dimensional speckle tracking echocardiography. PLoS One. 2017:12:e0180584

\section{Publisher's Note}

Springer Nature remains neutral with regard to jurisdictional claims in published maps and institutional affiliations.

Ready to submit your research? Choose BMC and benefit from:

- fast, convenient online submission

- thorough peer review by experienced researchers in your field

- rapid publication on acceptance

- support for research data, including large and complex data types

- gold Open Access which fosters wider collaboration and increased citations

- maximum visibility for your research: over $100 \mathrm{M}$ website views per year

At BMC, research is always in progress.

Learn more biomedcentral.com/submissions 